\title{
Arabic translation, adaptation and modification of the dialysis symptom index for chronic kidney disease stages four and five
}

Hayfa Almutary ${ }^{1,2^{*}}$, Ann Bonner ${ }^{1,3,4}$ and Clint Douglas ${ }^{1}$

\begin{abstract}
Background: Symptom burden in chronic kidney disease (CKD) is poorly understood. To date, the majority of research focuses on single symptoms and there is a lack of suitable multidimensional symptom measures. The purpose of this study was to modify, translate, cross-culturally adapt and psychometrically analyse the Dialysis Symptom Index (DSI).
\end{abstract}

Methods: The study methods involved four phases: modification, translation, pilot-testing with a bilingual non-CKD sample and then psychometric testing with the target population. Content validity was assessed using an expert panel. Inter-rater agreement, test-retest reliability and Cronbach's alpha coefficient were calculated to demonstrate reliability of the modified DSI. Discriminative and convergent validity were assessed to demonstrate construct validity.

Results: Content validity index during translation was 0.98 . In the pilot study with 25 bilingual students a moderate to perfect agreement (Kappa statistic $=0.60-1.00$ ) was found between English and Arabic versions of the modified DSI. The main study recruited 433 patients CKD with stages 4 and 5 . The modified DSI was able to discriminate between non-dialysis and dialysis groups $(p<0.001)$ and demonstrated convergent validity with domains of the Kidney Disease Quality of Life short form. Excellent test-retest and internal consistency (Cronbach's $a=0.91$ ) reliability were also demonstrated.

Conclusion: The Arabic version of the modified DSI demonstrated good psychometric properties, measures the multidimensional nature of symptoms and can be used to assess symptom burden at different stages of CKD. The modified instrument, renamed the CKD Symptom Burden Index (CKD-SBI), should encourage greater clinical and research attention to symptom burden in CKD.

Keywords: Chronic kidney disease, Symptom burden, Dialysis, Non-dialysis, Instrument

\section{Background}

Chronic kidney disease (CKD) is associated with a wide range of physical and psychological symptoms which have a negative impact on health-related quality of life [1]. Fatigue, pain, pruritus, sleep disturbances and decreased appetite are some of the most prevalent and frequently reported symptoms in CKD patients [2] and are the main predictors of increased morbidity and mortality $[3,4]$. Alleviating or controlling symptoms in CKD patients are one of the main priorities of the renal multidisciplinary team

\footnotetext{
* Correspondence: hayfa.almutary@connect.qut.edu.au

'School of Nursing, Queensland University of Technology, Victoria Park Rd, Kelvin Grove, Brisbane, QLD 4059, Australia

${ }^{2}$ School of Nursing, King Abdulaziz University, Jeddah, Saudi Arabia

Full list of author information is available at the end of the article
}

and thus require comprehensive assessment [5]. A major impediment to understanding the symptom experience in CKD is the lack of suitable measures that examine a wide range of symptoms across key dimensions (i.e. prevalence, severity, frequency and distress). Symptom prevalence alone does not reflect the complete patient symptom experience. The symptom experience is much better understood in cancer patients as a multidimensional burden comprising prevalence, distress, severity and frequency $[6,7]$. Though CKD patients often have greater prevalence and distress than advanced cancer patients [2], severity and frequency are rarely measured.

The Dialysis Symptom Index (DSI) is the most frequently used measure to assess symptoms in CKD and end stage 
kidney disease [2]. Developed by Weisbord et al. [8], the DSI was designed to examine the prevalence and distress of 30 self-reported physical and emotional symptoms. This contrasts with the purposes of other available instruments, such as the Modified Edmonton Symptom Assessment System [9], the Patient Outcome Scale Symptom Module (POS-S) [10] and the Pittsburgh Symptom Score [11] that have been used to assess symptom burden in CKD patients. However, none of the currently available instruments measure the multidimensional nature of symptoms $[12,13]$.

The ideal CKD symptom assessment measure should assess a range of symptoms and be able to determine the prevalence, distress, severity and frequency of symptoms [2]. The purpose of this study was to modify, translate, culturally adapt, and then evaluate the psychometric properties of the Arabic version of the DSI. It was part of a larger study examining symptom clusters in people with CKD.

\section{Methods}

The psychometric property testing involved four phases: modification of the DSI, translation, pilot study and the main study.

\section{Phase 1 - Instrument modification}

Permission was granted by Weisbord to modify and then translate the DSI into Arabic. First, two symptoms (depression and nocturia) and three free fields were added. Both depression [14] and nocturia [15,16] are highly prevalent in CKD, but are not routinely included in symptom measures. The additional free fields were included to capture any possible symptoms that were missing from the DSI. Then two symptom dimensions (severity and frequency) were also added to the DSI $[2,12,13]$. All symptom dimensions (frequency, severity and distress) were rated on a $0-10$ point scale because this type of response scale is easier for patients to use (i.e. similar to the commonly used numerical pain rating scale), provides a wider range of possible scores, and increases the statistical analyses that are available. In the original DSI, patients were asked to consider symptoms they had experienced in the previous week. However, we extended the time frame to four weeks to reflect the chronic nature of CKD symptoms. For example, some symptoms may occur often only during dialysis (e.g. cramps and dizziness) and may not be persistent over 4 weeks. These modifications created a comprehensive instrument, re-named (with permission from Weisbord) the CKD Symptom Burden Index (CKD-SBI), to assess symptoms in different stages of CKD. The time needed to complete the modified instrument ranged between 15 to 30 minutes.

The CKD-SBI includes four symptom dimensions (prevalence, distress, severity and frequency) across 32 symptoms (see Additional file 1). The prevalence scale assesses the presence or absence (Yes/No) of each symptom with a higher overall score indicating a higher symptom occurrence. The possible range of prevalence scores is 0-32. Distress, severity and frequency are each measured using a 0 to 10 scale for each of the symptoms. Participants rate the "distress" subscale from none to very much, "severity" from none to very severe, and "frequency" from never to constant. A maximum total score for each of these scales is 320 (range 0-320). The total CKD-SBI score is calculated by summing the scores of each CKD-SBI subscale (prevalence, distress, severity and frequency) and then multiplying the result by 0.1008 (constant number). The total score for the CKD-SBI scale therefore ranges between 0-100, where 100 indicates the highest symptom burden possible.

\section{Phase 2 - Translation procedure}

We used the Brislin back-translation method [14,17]; the most frequently used and accepted method for effective translation equivalence of instruments [18]. The method begins with a bilingual expert translating the instrument from its source language (English) into the target language (Arabic). Consultations with an expert panel are an important stage to ensure clarity, detect linguistic mistakes and ensure cross-cultural equivalence [19]. Then a blind back-translation (without accessing the original source language version) from the target language to the source language should be undertaken by another bilingual translator [18]. The back-translated version is then compared with the original version. If there are errors in the meaning in the blind back-translated version after comparison with the original version, the process of translation is repeated for the sections that include errors in the meaning [20]. Figure 1 summarises the steps in the Brislin's method.

Translation processes were undertaken in Saudi Arabia using bilingual (Arabic/ English), certified translators. Formal written Arabic language was used as this form of Arabic is used in all Arabic-speaking countries; thus increasing the usefulness of the instrument to other CKD populations outside of Saudi Arabia. The Arabic expert panel involved three nephrology nurses, two nephrologists, a social worker and two primary school teachers. The goal of the panel was to: 1) ensure clarity, readability and linguistic appropriateness; 2) review of grammatical style and comprehensibility; and 3) maintain cultural equivalence. The primary school teachers ensured suitable literacy level of the CKD-SBI for people with limited literacy skills (which will be tested in the pilot study). The expert panel recommended several minor linguistic and grammatical modifications, correction of spelling, and clearer instruction statements. A blind back-translation from the Arabic version into English was then performed by another bilingual certified translator independently 


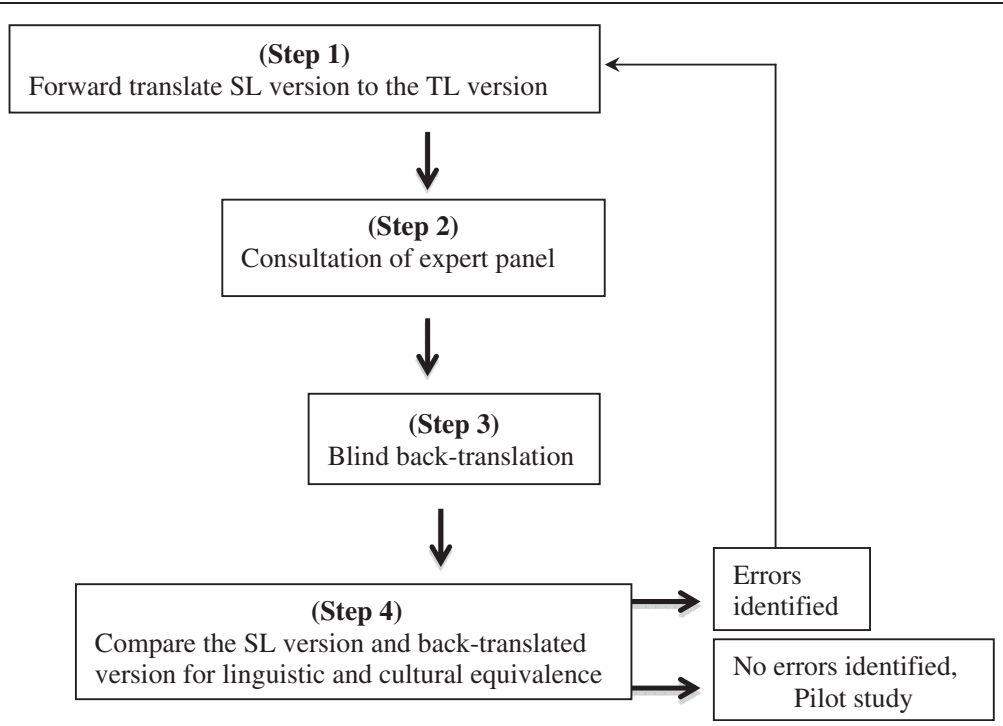

Figure 1 Steps of Brislin's method of translation application.

of the first translator and without access to the original English CKD-SBI. The procedure that was used to compare the back-translated version with the original version involved a new independent professional translator, two bilingual members of the expert panel and the first author. There were no errors identified in meaning after comparing both versions.

Content validity index (CVI) testing also occurred during this phase using the expert panel and a professional translator from a Saudi hospital. Individually they were asked to rate their level of agreement with the relevance and equivalence of each item between the Arabic and English versions on a four-point scale ( $1=$ 'not relevant' to $4=$ 'very relevant') to evaluate content validity [21]. A score of 3 or 4 indicates that the translated item is valid and equivalent to the English version [21]. The total CVI was 0.98 with items ranging from $0.88-1.00$, which indicated that the CKD-SBI had good content validity.

\section{Phase 3 - Pilot study}

Siaki [22] recommends pretesting of a translated instrument as a method to determine equivalence between the target language and source language. We conducted a pilot study of both versions of the CKD-SBI using bilingual university students and measured test-retest reliability, intra-rater reliability and internal consistency. As symptoms are also experienced by normal healthy populations but in a lesser frequency than those with CKD, using university students was appropriate when assessing language equivalence. Several studies have used this method when assessing language equivalence [23-25]. The pilot study involved anonymous completion of the English and Arabic versions of the CKD-SBI, on two separate occasions, one week apart. Demographic information (i.e. age, gender) was not relevant to determining equivalence and was not collected. An Arabic announcement was applied to the university notice board, and a snowball sampling technique was used to recruit participants during April-May, 2013. Ethics approval was obtained from the Queensland University of Technology (QUT) prior to commencement.

\section{Phase 4 - Main study}

The main study used a cross-sectional design to evaluate the psychometric properties of the CKD-SBI in Arabicspeaking CKD patients who were either receiving dialysis or not. A convenience sample of CKD patients from three hospitals in Saudi Arabia were recruited during July 2013 to February 2014. Initially treating clinicians identified potential eligible participants - adults with CKD (eGFR $<30$ $\mathrm{mls} / \mathrm{min} / 1.73 \mathrm{~m}^{2}$ ) or currently receiving either haemodialysis or peritoneal dialysis, willing to participate, and able to communicate in Arabic. Exclusion criteria were cognitive impairment that would preclude voluntary, informed consent, and those with critical conditions. Ethical approval was obtained from King Abdulaziz University Hospital, Jeddah Research Centre, and QUT Human Research Ethics Committees. All participants provided written informed consent. Data was collected through in-person distribution in the nephrology clinics (non-dialysis CKD patients) and the kidney dialysis centres (dialysis patients). Participants completed the newly translated Arabic version of the CKD-SBI (32 items) along with the Kidney Disease Quality of Life short form (KDQOL-36 ${ }^{\mathrm{Tm}}$ ). The KDQOL-36 ${ }^{\mathrm{Tm}}$ has 36 items and is available in Arabic [26]. The total score of the KDQOL-36 ranges from 0-100, with higher scores indicating a better QOL. Demographic and renal characteristics were also collected. 
Davies et al.'s [27] co-morbidity index was used to estimate the number of co-morbidities for the sample. This index consists of seven domains of co-morbid conditions, including malignancy (active, non-cutaneous); ischaemic heart disease, peripheral vascular disease, left ventricular dysfunction, diabetes mellitus (type 1 or 2); systemic collagen vascular disease; or other significant pathology. The total score is determined by summing the score of each co-morbid domain. The scores are then graded into three risk groups: grade 0 is a zero score, grade 1 (1-2 domains), or grade 2 (3-7 domains).

\section{Statistical analysis}

Data for the pilot study and the main study were entered into IBM SPSS Statistics version 21 in two different data sets and reported in the results section separately. For the pilot study, the percentage of agreement between the original English and Arabic versions of the CKD-SBI was calculated to examine equivalence. Kappa statistics were used to assess the inter-rater agreement between the English and Arabic versions for categorical data. Intra Class Correlation coefficient and Wilcoxon signed-ranks test for matched pairs (numerical scales) were used to determine the consistency of CKD-SBI scores. The ICC coefficient was used to estimate the inter-rater reliability of the CKD-SBI. Pearson's correlation coefficients were also calculated to assess the test-retest reliability. Cronbach's alpha reliability coefficients were calculated to determine the internal consistency.

For the main study, descriptive statistics were used to summarise sample characteristics. Cronbach's alpha reliability coefficients were calculated to determine the internal consistency of the CKD-SBI. Pearson's correlations were also used to examine associations between symptom dimensions. Discriminative validity was assessed using Welch $\mathrm{t}$-tests to assess if the CKD-SBI was able to discern between known groups according to disease severity within the CKD population. For convergent validity, Pearson correlation coefficients were used to examine the relationships between the symptom subscales of the CKD-SBI and all domains of KDQOL-36 ${ }^{\mathrm{m}}$. Significance was set at $p<0.05$.

\section{Results}

\section{Pilot study}

The pilot study involved 25 bilingual students, producing 25 matched English (E)/Arabic (A) pairs. There was a high percentage of agreement between $\mathrm{E}$ and $\mathrm{A}$ versions across all the items with a mean of $98.45 \pm 3.04$ (range $=$ 88-100\%). Level of agreement between the $\mathrm{E}$ and A versions revealed Kappa (к) scores ranging from $0.603-1.0$. The strength of agreement is considered poor when $\mathrm{K}<$ 0.20 , fair between 0.21 and 0.40 , moderate between 0.41 and 0.60 , substantial between 0.61 and 0.80 , and excellent when $\mathrm{k}>0.80$ [28]. Most items (23 out of 31) revealed a $\mathrm{K}$ of 1.00 indicating complete agreement (see Table 1). The ICC score indicated an excellent agreement between the $\mathrm{E}$ and A versions of the CKD-SBI (ICC $=0.99, p<0.001)$. In addition, the Wilcoxon signed rank test was used to examine the difference between the $\mathrm{E}$ and $\mathrm{A}$ versions for the total CKD-SBI scale and for each subscale (prevalence, distress, severity and frequency). The results revealed no significant differences between the $\mathrm{E}$ and $\mathrm{A}$ versions of the CKD-SBI for the total scale of CKD-SBI $(\mathrm{Z}=1.79, p=$

Table 1 Percentage of equivalence and Kappa statistics for English and Arabic CKD Symptom Burden Index for the pilot study $(N=25)$

\begin{tabular}{|c|c|c|}
\hline Symptom & $\begin{array}{l}\% \text { of } \\
\text { agreement }\end{array}$ & $\mathrm{K}(p)$ \\
\hline 1. Constipation & 92 & $0.841(<0.001)$ \\
\hline 2. Nausea & 100 & $1.00(<0.001)$ \\
\hline 3. Vomiting & 96 & $0.896(<0.001)$ \\
\hline 4. Diarrhoea & 100 & $1.00(<0.001)$ \\
\hline 5. Decreased appetite & 100 & $1.00(<0.001)$ \\
\hline 6. Muscle cramps & 100 & $1.00(<0.001)$ \\
\hline 7. Swelling in legs & 100 & $1.00(<0.001)$ \\
\hline 8. Shortness of breath & 100 & $1.00(<0.001)$ \\
\hline 9. Light headedness or dizziness & 100 & $1.00(<0.001)$ \\
\hline $\begin{array}{l}\text { 10. Restless legs or difficulty keeping } \\
\text { your legs still }\end{array}$ & 96 & $0.834(<0.001)$ \\
\hline 11. Numbness or tingling in feet & 100 & $1.00(<0.001)$ \\
\hline 12. Feeling tired or lack of energy & 100 & $1.00(<0.001)$ \\
\hline 13. Cough & 100 & $1.00(<0.001)$ \\
\hline 14. Dry mouth & 100 & $1.00(<0.001)$ \\
\hline 15. Bone or joint pain & 100 & $1.00(<0.001)$ \\
\hline 16. Chest pain & 100 & $1.00(<0.001)$ \\
\hline 17. Headache & 96 & $0.779(<0.001)$ \\
\hline 18. Muscle soreness & 100 & $1.00(<0.001)$ \\
\hline 19. Difficulty concentrating & 100 & $1.00(<0.001)$ \\
\hline 20. Dry skin & 100 & $1.00(<0.001)$ \\
\hline 21. Itching & 100 & $1.00(<0.001)$ \\
\hline 22. Worrying & 96 & $0.803(<0.001)$ \\
\hline 23. Feeling nervous & 96 & $0.826(<0.001)$ \\
\hline 24. Trouble falling asleep & 92 & $0.828(<0.001)$ \\
\hline 25. Trouble staying asleep & 100 & $1.00(<0.001)$ \\
\hline 26. Feeling irritable & 100 & $1.00(<0.001)$ \\
\hline 27. Feeling sad & 100 & $1.00(<0.001)$ \\
\hline 28. Feeling anxious & 88 & $0.603(0.001)$ \\
\hline 29. Depression & 100 & $1.00(<0.001)$ \\
\hline 30. Decreased interest in sex & 100 & $1.00(<0.001)$ \\
\hline 31. Difficulty becoming sexually aroused & 100 & $1.00(<0.001)$ \\
\hline 32. Nocturia & 100 & $1.00(<0.001)$ \\
\hline
\end{tabular}


$0.07)$, as well as for each subscale: prevalence $(Z=1.34$, $p=0.18)$, distress $(Z=1.14, p=0.26)$, severity $(Z=1.83$, $p=0.68)$ and frequency $(Z=1.48, p=0.14)$.

Pearson's correlation coefficients assessed test-retest reliability and revealed a strong positive correlation between the mean scores for both versions of the CKD-SBI $(r=0.997, p<0.01)$. A Cronbach's alpha coefficient of 0.7 is generally accepted to estimate the internal consistency of a new scale [29]. Cronbach's alpha coefficients for the E version (Cronbach's $\alpha=0.897$ ) and the A version (Cronbach's $\alpha=0.899$ ) both demonstrated good internal consistency.

\section{Main study}

\section{Sample characteristics}

A total of 433 patients with CKD were recruited. The mean age was $48.23 \pm 14.89$ years, $52.9 \%$ were male, and $58.4 \%$ had at least one co-morbid condition. Most of the participants were receiving dialysis treatment $(n=329$, $76 \%)$. Diabetes and hypertension were the leading causes of CKD in our sample. The overall mean of KDQOL-36 score was $58.08 \pm 20.04$, ranging from 6.88 to 99 . Sample characteristics are summarised in Table 2.

\section{CKD-SBI psychometric results}

The total mean CKD-SBI score was $17.98 \pm 16.11$, ranging from 0 to 83.36 . Symptoms were highly prevalent in this sample of CKD patients (mean $=12.58 \pm 7.9$; range $0-32$ ).

Table 2 Sample characteristics for the main study $(N=433)$

\begin{tabular}{lll}
\hline Characteristics & $\boldsymbol{n}$ & \% \\
\hline Age (mean, SD) & 48.23 & 14.89 \\
Gender & & \\
Male & 229 & 52.9 \\
Female & 204 & 47.1 \\
Marital status & & \\
Married & 257 & 59.4 \\
Not married & 175 & 40.4 \\
Treatment type & & \\
Dialysis & 329 & 76 \\
Non-dialysis (eGFR 5-29 mL/min/1.73 m²) & 104 & 24 \\
Causes of CKD & & \\
Diabetic nephropathy & 135 & 31.2 \\
Hypertensive nephropathy & 146 & 33.7 \\
Primary glomerular disease & 30 & 6.9 \\
Unknown aetiology & 59 & 13.6 \\
Others & 63 & 14.5 \\
Comorbid conditions & & \\
0 & 180 & 41.6 \\
1-2 & 223 & 51.5 \\
$\leq 3$ & 30 & 6.9 \\
\hline
\end{tabular}

Percentages vary depending on missing data.
As the purpose of this article is to present the psychometric analysis of the CKD-SBI further descriptive results are not required and will be presented in another separate paper. Summary statistics for each subscale of CKD-SBI are presented in Table 3. There was a high internal consistency of the overall CKD-SBI (Cronbach's $\alpha=$ 0.91 ) and subscales (range $=0.93-0.90$ ). There were strong positive intercorrelations between CKD-SBI subscales, which ranged from 0.82 - 0.97 (see Table 3).

\section{Discriminative validity}

Discriminative validity is the ability of an instrument to distinguish between groups that are expected to differ based on their clinical diagnosis or other characteristics [30]. It is an important measure of construct validity, which is commonly examined through the "known groups" technique [30]. As renal function deteriorates, symptoms become more prevalent due to the accumulation of uraemic toxins [31]. Thus we divided the sample into two groups (dialysis and non-dialysis patients) and compared the total symptom burden score. We hypothesised that symptom burden would increase as GFR decreases. Due to the unequal sample size, Welch t-tests were used to compare groups [32]. This showed significant differences in total symptom burden scores $(t(403.5)=-13.1, p<0.001)$, indicating that the CKD-SBI is able to discriminate between groups that have different eGFR levels.

\section{Convergent validity}

Convergent validity refers to the degree to which scores on a measure associate with scores on other measures that are intended to assess similar constructs [33]. Previous studies have shown that symptoms among CKD patients are correlated with poor health-related quality of life $[34,35]$. Therefore, we hypothesised that all symptom dimension scores would be negatively correlated with domain scores of the KDQOL-36. Pearson's correlation coefficients demonstrated moderate to strong relationships in the expected direction between all symptom dimensions (prevalence, distress, severity and frequency) and each domain of the KDQOL-36 (see Table 4).

\section{Discussion}

This study modified, translated, culturally adapted and psychometrically evaluated the CKD-SBI. Jablonski [12] argues that when examining CKD symptom burden, the multidimensional nature of symptoms should be considered. However, a significant criticism of research in this area is the lack of comprehensive CKD symptom measures. The DSI was selected as a target instrument in this study for two reasons. First, there is no disease-specific instrument in Arabic for assessing CKD symptoms. Second, we believed that the DSI is the best available instrument for symptom assessment but it required some modifications to 
Table 3 Descriptive statistics, reliability and Pearson's correlation coefficient of the CKD-SBI subscales

\begin{tabular}{lllllllll}
\hline Subscale & Mean & SD & Range & $\mathbf{a}$ & $\mathbf{1}$ & $\mathbf{2}$ & $\mathbf{3}$ & $\mathbf{4}$ \\
\hline 1. Prevalence & 12.58 & 7.89 & $0-32$ & 0.924 & - & & & \\
2. Distress & 55.47 & 51.74 & $0-259$ & 0.904 & 0.817 & - & & \\
3. Severity & 57.56 & 52.26 & $0-265$ & 0.924 & 0.835 & 0.963 & - & 0.967 \\
4. Frequency & 58.76 & 50.48 & $0-273$ & 0.929 & 0.838 & 0.943 & - & 0.991 \\
5. Total symptom burden & 17.84 & 15.98 & $0-83$ & 0.91 & 0.857 & 0.981 & 0.983 & - \\
\hline
\end{tabular}

be more comprehensive to assess the multidimensional aspects of symptoms and gain better understanding of symptom burden.

The results demonstrated that the CKD-SBI was linguistically and culturally relevant to CKD patients from Arabic-speaking backgrounds. In the translation process, no statements required adjustment to fit into the Arabic cultural context. Excellent content validity of the Arabic version of the CKD-SBI was demonstrated. Since formal written Arabic language is universally understood amongst Arabic speaking nations, this instrument is applicable for other populations. The DSI was originally developed and validated for patients receiving dialysis treatment. After modification, testing and with approval from the DSI originator, we renamed the DSI as the CKD Symptom Burden Index (CKD-SBI), to reflect the utility of the instrument to measure symptom burden and to broaden its appeal to other researchers to assess symptoms in the non-dialysis CKD populations.

In this study, symptoms were highly prevalent among CKD patients with an average of 12.58 symptoms per patient. This finding is consistent with a recent comprehensive review of 19 studies, which found the mean symptom per patients ranged between 6-20 symptoms [2]. Based on literature, symptoms in CKD were often described as a part of KDQOL [2]. However, the KDQOL does not measure the most prevalent symptoms reported in the literature; that is fatigue, bone or joint pain, and psychological symptoms. This suggests that using the symptom list of the KDQOL is not sufficient measure symptom burden in CKD patients. The results from this study emphasise using a comprehensive symptom measure to examine symptom burden among CKD patients.

Overall the results provide initial evidence of validity and reliability of the CKD-SBI, which has potential clinical and research implications. One of the strengths of the main study was having a large sample size and recruiting CKD patients from three different hospitals. Sample characteristics in this study were similar to population data reported by the Saudi Center for Organ Transplantation [36] and a recent review on CKD in Saudi Arabia [37], which provides some evidence that participants were representative of the target population. We also administered the scale to patients with different treatment modalities and stages of CKD. Good discriminant validity was demonstrated, indicating that the CKD-SBI can discriminate between patients in stages 4 and 5 . A consistent technique (in-person distribution by one person [first author]) was used to administer the instrument. This technique assisted with providing insight into the clinical utility of the instrument. Patients reported acceptability and clarity of the instrument regardless of age and educational level. The time taken to complete it was short (on average 20 minutes) although this was dependent on the number of symptoms experienced.

Further evidence of construct validity was demonstrated by examining relationships between the CKD-SBI and domains of KDQOL-36. These results are consistent with previous studies that have demonstrated CKD symptoms are negatively correlated with KDQOL $[9,13,35]$. Similarly, Abdel-kader et al. [34] found moderate correlation between the DSI and Physical Component Summary and

Table 4 Pearson correlations between CKD-SBI symptom dimension subscales and KDQOL-36 domains $(N=433)$

\begin{tabular}{|c|c|c|c|c|c|c|}
\hline \multirow[t]{2}{*}{ CKD-SBI } & \multicolumn{6}{|l|}{ KDQOL-36 } \\
\hline & $\begin{array}{l}\text { Total KDQOL } \\
\text { scale score }\end{array}$ & $\begin{array}{l}\text { Physical function } \\
\text { summary }\end{array}$ & $\begin{array}{l}\text { Mental health } \\
\text { summary }\end{array}$ & $\begin{array}{l}\text { Effects of kidney } \\
\text { disease on daily life }\end{array}$ & $\begin{array}{l}\text { Symptoms and } \\
\text { problems subscale* }\end{array}$ & $\begin{array}{l}\text { Burden of } \\
\text { the disease }\end{array}$ \\
\hline Prevalence & $r=-0.58^{* *}$ & $r=-0.51^{* *}$ & $r=-0.41^{* *}$ & $r=-0.47^{* *}$ & $r=0.72^{* *}$ & $r=-0.33^{* *}$ \\
\hline Distress & $r=-0.63^{* *}$ & $r=-0.51^{* *}$ & $r=-0.44^{* *}$ & $r=-0.54^{* *}$ & $r=0.78^{* *}$ & $r=-0.36^{* *}$ \\
\hline Severity & $r=-0.61^{* *}$ & $r=-0.49^{* *}$ & $r=-0.43^{* *}$ & $r=-0.51^{* *}$ & $r=0.77^{* *}$ & $r=-0.34^{* *}$ \\
\hline Frequency & $r=-0.61^{* *}$ & $r=-0.51^{* *}$ & $r=-0.44^{* *}$ & $r=-0.49^{* *}$ & $r=0.75^{* *}$ & $r=-0.35^{* *}$ \\
\hline Total symptom burden & $r=-0.63^{* *}$ & $r=-0.52^{* *}$ & $r=-0.44^{* *}$ & $r=-0.53^{* *}$ & $r=0.75^{* *}$ & $r=-0.44^{* *}$ \\
\hline
\end{tabular}

*The results are presented after reverse scoring the symptoms and problems subscale of the KDQOL-36 to be consistent and in the same direction with our scoring system.

${ }^{* *}$ Correlation is significant at the 0.01 level (2-tailed). 
Mental Component Summary of the quality of life domains using the Medical Outcomes Study Short Form-36. The results suggest that the two instruments, CKD-SBI and KDQOL-36, together demonstrated convergent validity. Used together, they provide clinicians with a comprehensive understanding of CKD symptom burden and relationship with quality of life. The additional information gained from this new multidimensional instrument is better to predict the important patient outcomes such as HRQoL, depression, morbidity, mortality, which is worth the additional respondent burden. However, further work is needed to demonstrate the strong relationships between symptom burden and other important measures of HRQOL, depression, morbidity, and mortality.

\section{Limitations}

The results are significant as this is the first study to validate a comprehensive instrument designed to assess CKD symptom burden. However, there are several limitations that should be acknowledged. It is difficult to identify bilingual patients thus test-retest agreement may have been overestimated by use of the instrument in a generally healthy population (university students). Also, the testretest reliability was one week apart, thus there was potential for stability bias as participants could have memorised their responses between the two tests [30]. Since this instrument has been validated only in Arabic-speaking people with CKD stage 4 and 5, more research is needed to validate this instrument in English-speaking populations as well as in other CKD stages. In addition, it would have been ideal to compare the CKD-SBI with another multidimensional symptom measure, but another suitable instrument is not yet available. This limits the establishment of criterion-related validity for the CKD-SBI.

\section{Conclusion}

The CKD-SBI demonstrated good psychometric properties and is suitable to assess symptom burden for different CKD populations. It is comprehensive and can capture the multidimensional aspects of a range of symptoms. Assessing symptom burden could help health professionals to have a better understanding of the symptom experience of CKD patients and to identify symptoms that have a major impact on health-related quality of life. The lack of knowledge of the symptom experience and its impact on people's lives leads to under-assessment and treatment of symptoms. This new multidimensional instrument should encourage greater clinical and research attention to symptom burden in CKD.

\section{Additional file}

Additional file 1: Arabic version of CKD-SBI.
Competing interests

The authors declare that they have no competing interests.

\section{Authors' contributions}

Each of the co-authors has contributed significantly. HA designed the study, conducted data collection, statistical analyses and drafted the manuscript. $A B, C D$ participated in the research design and made critical revisions to the manuscript. All authors approved the final manuscript.

\section{Acknowledgements}

Doctoral scholarship provided by the Ministry of Higher Education, King Abdulaziz University, Saudi Arabia. We appreciate the permission afforded by Dr Weisbord to modify, translate and rename the Dialysis Symptom Index in this study.

\section{Author details}

${ }^{1}$ School of Nursing, Queensland University of Technology, Victoria Park Rd, Kelvin Grove, Brisbane, QLD 4059, Australia. ${ }^{2}$ School of Nursing, King Abdulaziz University, Jeddah, Saudi Arabia. ${ }^{3}$ Chronic Kidney Disease Centre for Research Excellence, University of Queensland, Brisbane, Australia. ${ }^{4}$ Visiting Research Fellow, Renal Medicine, Royal Brisbane and Women's Hospital, Brisbane, Australia.

Received: 17 October 2014 Accepted: 18 March 2015

Published online: 27 March 2015

\section{References}

1. lyasere O, Brown EA. Determinants of quality of life in advanced kidney disease: time to screen? Postgrad Med J. 2014;90(1064):340-7.

2. Almutary $H$, Bonner $A$, Douglas $C$. Symptom burden in chronic kidney disease: a review of recent literature. J Ren Care. 2013;39(3):140-50.

3. Amro A, Waldum B, Lippe N, Brekke FB, Dammen T, Miaskowski C, et al. Symptom clusters predict mortality among dialysis patients in Norway: a prospective observational cohort study. J Pain Symptom Manage. 2015;49(1):27-35.

4. Jhamb M, Argyropoulos C, Steel JL, Plantinga L, Wu AW, Fink NE, et al. Correlates and outcomes of fatigue among incident dialysis patients. Clin J Am Soc Nephrol. 2009;4(11):1779-86.

5. Murtagh F, Addington-Hall J, Higginson IJ. The prevalence of symptoms in end-stage renal disease: a systematic review. Adv Chronic Kidney Dis. 2007;14(1):82-99.

6. Cataldo JK, Paul S, Cooper B, Skerman H, Alexander K, Aouizerat B, et al. Differences in the symptom experience of older versus younger oncology outpatients: a cross-sectional study. BMC Cancer. 2013;13(1):1-16.

7. Pettersson G, Berterö C, Unosson M, Börjeson S. Symptom prevalence, frequency, severity, and distress during chemotherapy for patients with colorectal cancer. Support Care Cancer. 2014;22(5):1171-9.

8. Weisbord S, Fried LF, Arnold RM, Rotondi AJ, Fine MJ, Levenson DJ, et al. Development of a symptom assessment instrument for chronic hemodialysis patients: The Dialysis Symptom Index. J Pain Symptom Manage. 2004;27(3):226-40.

9. Davison $\mathrm{S}$, Jhangri $\mathrm{G}$, Johnson J. Cross-sectional validity of a modified Edmonton symptom assessment system in dialysis patients: a simple assessment of symptom burden. Kidney Int. 2006;69(9):1621-5.

10. Murphy EL, Murtagh FEM, Carey I, Sheerin NS. Understanding symptoms in patients with advanced chronic kidney disease managed without dialysis: use of a short patient-completed assessment tool. Nephron Clin Pract. 2009;111(1):C74-80.

11. Novak MJ, Sheth H, Bender FH, Fried L, Piraino B. Improvement in Pittsburgh Symptom Score index after initiation of peritoneal dialysis. Adv Perit Dial. 2008;24:46-50.

12. Jablonski A. The multidimensional characteristics of symptoms reported by patients on hemodialysis. Nephrol Nurs J. 2007;34(1):29-38.

13. Danquah F, Zimmerman L, Diamond P, Meininger J, Bergstrom N. Frequency, severity, and distress of dialysis-related symptoms reported by patients on hemodialysis. Nephrol Nurs J. 2010;37(6):627-38.

14. Palmer S, Vecchio M, Craig JC, Tonelli M, Johnson DW, Nicolucci A, et al. Prevalence of depression in chronic kidney disease: systematic review and meta-analysis of observational studies. Kidney Int. 2013;84(1):179-91. 
15. Wu M-Y, Wu Y-L, Hsu Y-H, Lin Y-F, Fan Y-C, Lin Y-C, et al. Risks of nocturia in patients with chronic kidney disease-do the metabolic syndrome and its components matter? J Urol. 2012;188(6):2269-73.

16. Hsu CY, Iribarren C, McCulloch CE, Darbinian J, Go AS. Risk factors for end-stage renal disease: 25-year follow-up. Arch Intern Med. 2009;169(4):342-50.

17. Brislin RW. Back-translation for cross-cultural research. J Cross-Cultural Psychology. 1970;1(3):185-216.

18. Eun-Seok C, Kim KH, Erlen JA. Translation of scales in cross-cultural research: issues and techniques. J Adv Nurs. 2007;58(4):386-95.

19. Chaboyer W, Lee B-O, Wallis M, Chien C-S. Taiwanese translation and psychometric testing of the revised illness perception questionnaire for patients with traumatic injury. J Clin Nurs. 2012;21(23/24):3466-74.

20. Jones PS, Lee JW, Phillips LR, Zhang XE, Jaceldo KB. An adaptation of Brislin's translation model for cross-cultural research. Nurs Res. 2001;50(5):300-4.

21. Tappen R. Advanced nursing research: from theory to practice. Sudbury, MA: Jones \& Bartlett Learning; 2011

22. Siaki LA. Translating questions for use with Samoan adults: lessons learned. J Transcult Nurs. 2011;22(2):122-8.

23. Bonner A, Wellard S, Kenrick M. Development and validation of the Human Activity Profile into Chinese language: lessons in determining equivalence. Nurs Health Sci. 2006;8(1):36-43.

24. Ghassemzadeh H, Mojtabai R, Karamghadiri N, Ebrahimkhani N. Psychometric properties of a Persian-language version of the Beck Depression Inventory-Second edition: BDI-II-PERSIAN. Depress Anxiety. 2005;21(4):185-92

25. Xinhu J, Xiyu C, Mingtian Z, Lingyu C, Xiongzhao Z, Shuqiao Y, et al. The psychometric properties of the Cognitive-Somatic Anxiety Questionnaire in Chinese undergraduate students and clinical patients. Compr Psychiatry. 2014;55(7):1751-6.

26. Al Jumaih A, Al-Onazi K, Binsalih S, Hejaili F, Al-Sayyari A. A study of quality of life and its determinants among hemodialysis patients using the KDQOL-SF instrument in one center in Saudi Arabia. Arab J Nephrol Transplant. 2011;4(3):125-30.

27. Davies SJ, Phillips L, Naish PF, Russell Gl. Quantifying comorbidity in peritoneal dialysis patients and its relationship to other predictors of survival. Nephrol Dial Transplant. 2002;17(6):1085-92.

28. Gisev N, Bell JS, Chen TF. Interrater agreement and interrater reliability: key concepts, approaches, and applications. Res Social Adm Pharm. 2013:9(3):330-8.

29. DeVon HA, Block ME, Moyle-Wright P, Ernst DM, Hayden SJ, Lazzara DJ, et al. A psychometric toolbox for testing validity and reliability. J Nurs Scholarsh. 2007:39(2):155-64.

30. Streiner D, Norman G. Health measurement scales. A practical guide to their development and use. 4th ed. New York: Oxford University Press; 2008.

31. Qaseem A, Hopkins Jr RH, Sweet DE, Starkey M, Shekelle P. Screening, monitoring, and treatment of stage 1 to 3 chronic kidney disease: a clinical practice guideline from the American College of Physicians. Ann Intern Med. 2013;159(12):835-47.

32. Keselman HJ, Othman AR, Wilcox RR, Fradette $K$. The new and improved two-sample $t$ test. Psychol Sci. 2004;15(1):47-51.

33. Carlson KD, Herdman AO. Understanding the impact of convergent validity on research results. Organizational Res Methods. 2012;15(1):17-32.

34. Abdel-Kader K, Unruh ML, Weisbord SD. Symptom burden, depression, and quality of life in chronic and end-stage kidney disease. Clin J Am Soc Nephrol. 2009:4(6):1057-64

35. Yong DSP, Kwok AOL, Wong DML, Suen MHP, Chen WT, Tse DMW. Symptom burden and quality of life in end-stage renal disease: a study of 179 patients on dialysis and palliative care. Palliat Med. 2009;23(2):111-9.

36. Saudi Center for Organ Transplantation (SCOT). Annual report 2013. Available from http://www.scot.org.sa/en/.

37. Almutary H, Bonner A, Douglas C. Chronic kidney disease in Saudi Arabia: a nursing perspective. Middle East J Nursing. 2013;7(6):17-26.

\section{Submit your next manuscript to BioMed Central and take full advantage of:}

- Convenient online submission

- Thorough peer review

- No space constraints or color figure charges

- Immediate publication on acceptance

- Inclusion in PubMed, CAS, Scopus and Google Scholar

- Research which is freely available for redistribution

Submit your manuscript at www.biomedcentral.com/submit 